\title{
A MINIMIZATION THEOREM IN QUASI-METRIC SPACES AND ITS APPLICATIONS
}

\author{
JEONG SHEOK UME
}

Received 25 October 2001

\begin{abstract}
We prove a new minimization theorem in quasi-metric spaces, which improves the results of Takahashi (1993). Further, this theorem is used to generalize Caristi's fixed point theorem and Ekeland's $\varepsilon$-variational principle.
\end{abstract}

2000 Mathematics Subject Classification: 47H10.

1. Introduction. Caristi [1] proved a fixed point theorem on complete metric spaces which generalizes the Banach contraction principle. Ekeland [3] also obtained a nonconvex minimization theorem, often called the $\varepsilon$-variational principle, for a proper lower semicontinuous function, bounded from below, on complete metric spaces. Later Takahashi [4] proved the following minimization theorem: let $X$ be a complete metric space and let $f: X \rightarrow(-\infty, \infty]$ be a proper lower semicontinuous function, bounded from below. Suppose that, for each $u \in X$ with $f(u)>\inf _{x \in X} f(x)$, there exists $v \in X$ such that $v \neq u$ and $f(v)+d(u, v) \leq f(u)$. Then there exists $x_{0} \in X$ such that $f\left(x_{0}\right)=\inf _{x \in X} f(x)$. These theorems are very useful tools in nonlinear analysis, control theory, economic theory, and global analysis.

2. Main results. Throughout this note, we denote by $\mathbb{N}$ the set of all positive integers and by $\mathbb{R}$ the set of all real numbers.

DEFINITION 2.1. A real-valued function $\Phi$ defined on a topological space $X$ is said to be lower semicontinuous at $x$ in $X$ if and only if $\left\{x_{\lambda}\right\}$ is a net in $X$ and $\lim x_{\lambda}=x$ implies $\Phi x \leq \liminf \Phi x_{\lambda}$.

Definition 2.2 [2]. A real-valued function $\Phi$ defined on a topological space $X$ is said to be weak lower semicontinuous at $x \in X$ if and only if $\left\{x_{\lambda}\right\}$ is a net in $X$ and $\lim x_{\lambda}=x$ implies $\Phi x \leq \lim \sup \Phi x_{\lambda}$. A mapping $\Phi$ is said to be a weak lower semicontinuous on $X$ if and only if it is weak lower semicontinuous for every $x \in X$.

Definition 2.3. A pair $(X, d)$ of a set $X$ and a mapping $d$ from $X \times X$ into real numbers is said to be a quasi-metric space if and only if

$$
\begin{aligned}
& d(x, y) \geq 0, \quad d(x, y)=0 \quad \text { iff } x=y, \\
& d(x, z) \leq d(x, y)+d(y, z) \quad \forall x, y, z \in X .
\end{aligned}
$$


DEFINITION 2.4. A sequence $\left\{x_{n}\right\}$ in $X$ is said to be a left $k$-Cauchy sequence if for each $k \in \mathbb{N}$ there is an $N_{k}$ such that

$$
d\left(x_{n}, x_{m}\right)<\frac{1}{k} \quad \forall m \geq n \geq N_{k} .
$$

A quasi-metric space is left $k$-sequentially complete if each left $k$-Cauchy sequence is convergent.

THEOREM 2.5. Let $(X, d)$ be left $k$-sequentially complete quasi metric space such that for each $x \in X$ the mapping $u \rightarrow d(x, u)$ is a lower semicontinuous on $X$. Let $f: X \rightarrow(-\infty, \infty]$ be a proper weak lower semicontinuous function bounded from below such that for any $u \in X$ with $\inf _{x \in X} f(x)<f(u)$, there exists $v \in X$ with $v \neq u$ and $f(v)+d(u, v) \leq f(u)$. Then there exists $x_{0} \in X$ such that $\inf _{x \in X} f(x)=f\left(x_{0}\right)$.

Proof. Suppose that $\inf _{x \in X} f(x)<f(y)$ for every $y \in X$. For each $y \in X$, we define $S(y)$ by

$$
S(y)=\{z \in X: f(z)+d(y, z) \leq f(y)\} .
$$

From (2.3) and hypotheses of the theorem we have the following:

(*) For each $y \in X$, there exists $v \in X$ with $v \neq y$ such that $v \in S(y)$, and for each $z \in S(y), S(z) \subseteq S(y)$.

For each $y \in X$, we define $A(y)$ by

$$
A(y)=\inf \{f(z): z \in S(y)\} .
$$

Choose $u \in X$ with $f(u)<\infty$. Then we choose a sequence $\left\{u_{n}\right\}$ in $S(u)$ as follows: when $u=u_{1}, u_{2}, \ldots, u_{n}$ have been chosen, choose $u_{n+1} \in S\left(u_{n}\right)$ such that

$$
f\left(u_{n+1}\right)<A\left(u_{n}\right)+\frac{1}{n} .
$$

Thus, we obtain a sequence $\left\{u_{n}\right\}$ such that

$$
\begin{aligned}
& d\left(u_{n}, u_{n+1}\right) \leq f\left(u_{n}\right)-f\left(u_{n+1}\right), \\
& f\left(u_{n+1}\right)-\frac{1}{n}<A\left(u_{n}\right) \leq f\left(u_{n+1}\right) .
\end{aligned}
$$

By (2.6), $\left\{f\left(u_{n}\right)\right\}$ is a nonincreasing sequence of reals and so it converges. Therefore, by (2.7) there is some $\alpha$ in $\mathbb{R}$ such that

$$
\alpha=\lim _{n \rightarrow \infty} A\left(u_{n}\right)=\lim _{n \rightarrow \infty} f\left(u_{n}\right)=\inf _{n \in N} f\left(u_{n}\right) .
$$

Let $k \in \mathbb{N}$ be arbitrary. By (2.8) there exists some $N_{k}$ such that $f\left(u_{n}\right)<\alpha+1 / k$ for all $n \geq N_{k}$. Thus, by monotony of $\left\{f\left(u_{n}\right)\right\}$, for $m \geq n \geq N_{k}$, we have

$$
\alpha \leq f\left(u_{m}\right) \leq f\left(u_{n}\right)<\alpha+\frac{1}{k}
$$

and hence

$$
f\left(u_{n}\right)-f\left(u_{m}\right)<\frac{1}{k} \quad \forall m>n \geq N_{k}
$$


From the triangle inequality, (2.6) and (2.10), we get

$$
\begin{aligned}
d\left(u_{n}, u_{m}\right) & \leq \sum_{i=n}^{m-1} d\left(u_{i}, u_{i+1}\right) \leq \sum_{i=n}^{m-1}\left[f\left(u_{i}\right)-f\left(u_{i+1}\right)\right] \\
& \leq f\left(u_{n}\right)-f\left(u_{m}\right)<\frac{1}{k}
\end{aligned}
$$

for all $m>n \geq N_{k}$.

Therefore, $\left\{u_{n}\right\}$ is a left $k$-Cauchy sequence in $X$. By completeness, there exists $z \in X$ such that $u_{n} \rightarrow z$. Since $f$ is a weak lower semicontinuous; by (2.8), we have

$$
f(z) \leq \limsup _{n \rightarrow \infty} f\left(u_{n}\right)=\alpha .
$$

From (2.11), we obtain

$$
f\left(u_{m}\right) \leq f\left(u_{n}\right)-d\left(u_{n}, u_{m}\right) .
$$

Since $f$ is a weak lower semicontinuous on $X$ and $u \rightarrow d(x, u)$ on $X$ is a lower semicontinuous, we have

$$
\begin{aligned}
f(z) & \leq \limsup _{m \rightarrow \infty} f\left(u_{m}\right) \leq f\left(u_{n}\right)+\limsup _{m \rightarrow \infty}\left[-d\left(u_{n}, u_{m}\right)\right] \\
& =f\left(u_{n}\right)-\liminf _{m \rightarrow \infty} d\left(u_{n}, u_{m}\right)=f\left(u_{n}\right)-d\left(u_{n}, z\right) .
\end{aligned}
$$

Hence

$$
d\left(u_{n}, z\right) \leq f\left(u_{n}\right)-f(z) .
$$

From (2.3) and (2.15), we obtain that $z \in S\left(u_{n}\right)$ for every $n \in \mathbb{N}$ and hence

$$
A\left(u_{n}\right) \leq f(z) \quad \forall n \in \mathbb{N} .
$$

Taking the limit when $n$ tends to infinity, we have

$$
\lim _{n \rightarrow \infty} A\left(u_{n}\right) \leq f(z) .
$$

From (2.8), (2.12), and (2.17), we have

$$
f(z)=\alpha .
$$

Since $z \in S\left(u_{n}\right)$ and $u_{n} \in S(u)$, by $(*)$, we obtain $z \in S(u)$. Suppose that $v_{1} \in S(z)$ and $v_{1} \neq z$. Then $f\left(v_{1}\right)<f(z)$ or by (2.18), $f\left(v_{1}\right)<\alpha$. Since $v_{1} \in S(z), z \in S\left(u_{n}\right)$ and $u_{n} \in S(u)$, by $(*)$, we have $S(z) \subseteq S\left(u_{n}\right) \subseteq S(u)$. Hence $v_{1} \in S\left(u_{n}\right)$ and $v_{1} \in S(u)$. Thus

$$
A\left(u_{n}\right) \leq f\left(v_{1}\right) \quad \forall n \in \mathbb{N} .
$$

Taking the limit when $n$ tends to infinity, we get

$$
\alpha \leq f\left(v_{1}\right) .
$$

This is in contradiction with $f\left(v_{1}\right)<\alpha$. Hence $S(z)=\{z\}$. But, by (2.3) and hypothesis of a function $f$ in theorem there exists $y \in X$ such that $y \neq z$ and $\{y, z\} \subseteq S(z)$. This is a contradiction. This completes the proof. 
REMARK 2.6. Theorem 2.5 is a generalization of Takahashi's minimization theorem [4].

THEOREM 2.7. Let $(X, d)$ be left $k$-sequentially complete quasi-metric space such that for each $x \in X$, the mapping $u \rightarrow d(x, u)$ is a lower semicontinuous on $X$. Let $f: X \rightarrow(-\infty, \infty]$ be a proper weak lower semicontinuous function bounded from below. Assume that there exists a selfmapping $T$ of $X$ such that

$$
f(T x)+d(x, T x) \leq f(x) \quad \forall x \in X
$$

Then $T$ has a fixed point in $X$.

Proof. Since $f$ is proper, there exists $v \in X$ such that $f(v)<\infty$. Put

$$
Z=\{x \in X \mid f(x) \leq f(v)\} .
$$

Then, since $f$ is weak lower semicontinuous, $Z$ is closed. So $Z$ is left $k$-sequentially complete. Let $x \in Z$. Then, Since

$$
f(T x)+d(x, T x) \leq f(x) \leq f(v) .
$$

So $Z$ is invariant under $T$. Assume that $T x \neq x$ for every $x \in Z$. Then by Theorem 2.5, there exists $u \in Z$ such that $f(u)=\inf _{x \in X} f(x)$. Since $f(T u)+d(u, T u) \leq f(u)$ and $f(u)=\inf _{x \in Z} f(x)$, we have $f(T u)=f(u)=\inf _{x \in Z} f(x)$ and $d(u, T u)=0$. Hence $T u=u$. This is a contradiction. Therefore $T$ has a fixed point $u$ in $Z$. This completes the proof.

REMARK 2.8. Theorem 2.7 is a generalization of Caristi's fixed point theorem [1].

The following theorem is a generalization of Ekeland's $\varepsilon$-variational principle [3].

THEOREM 2.9. Let $(X, d)$ be left $k$-sequentially complete quasi-metric space such that for each $x \in X$ the mapping $u \rightarrow d(x, u)$ is a lower semicontinuous on $X$. Let $f: X \rightarrow(-\infty, \infty]$ be a proper weak lower semicontinuous function bounded from below. Then,

(1) for any $u \in X$ with $f(u)<\infty$, there exists $v \in X$ such that $f(v) \leq f(u)$ and $f(w)>f(v)-d(v, w)$ for every $w \in X$ with $w \neq v$;

(2) for any $\varepsilon>0$ and $u \in X$ with $f(u)<\inf _{x \in X} f(x)+\varepsilon$, there exists $v \in X$ such that $f(v) \leq f(u), d(u, v) \leq 1$ and $f(w)>f(v)-\varepsilon d(v, w)$ for every $w \in X$ with $w \neq v$.

Proof. (1) Let $u \in X$ be such that $f(u)<\infty$ and let

$$
Y=\{x \in X \mid f(x) \leq f(u)\} .
$$

Then $Y$ is nonempty and complete. We prove that there exists $v \in Y$ such that $f(w)>f(v)-d(v, w)$ for every $w \in X$ with $w \neq v$. If not, for every $x \in Y$, there exists $w \in X$ such that $w \neq x$ and $f(w)+d(x, w) \leq f(x)$. Since $f(w) \leq f(x) \leq f(u), w \in X$ is an element of $Y$. By Theorem 2.5, there exists $x_{0} \in Y$ such that $f\left(x_{0}\right)=\inf _{x \in Y} f(x)$. For this $x_{0} \in Y$, there exists $x_{1} \in Y$ such that $x_{0} \neq x_{1}$ and $f\left(x_{1}\right)+d\left(x_{0}, x_{1}\right) \leq f\left(x_{0}\right)$. 
Thus we have $f\left(x_{0}\right)=f\left(x_{1}\right)$ and $d\left(x_{0}, x_{1}\right)=0$. Hence $x_{0}=x_{1}$. This is a contradiction. Therefore (1) holds.

(2) Put

$$
Z=\{x \in X \mid f(x) \leq f(u)-\varepsilon d(u, x)\} .
$$

Then $Z$ is nonempty and complete. Since $\varepsilon d(u, x)$ is a quasi metric, as in the proof of (1), we have that there exists $v \in Z$ such that $f(w)>f(v)-\varepsilon d(v, w)$ for every $w \in X$ with $w \neq v$. Since $v \in Z$, we have $f(v) \leq f(u)-\varepsilon d(u, v) \leq f(u)$ and

$$
d(u, v) \leq \frac{1}{\varepsilon}[f(u)-f(v)] \leq \frac{1}{\varepsilon}\left[f(u)-\inf _{x \in X} f(x)\right] \leq \frac{1}{\varepsilon} \cdot \varepsilon=1 .
$$

This completes the proof of (2).

REMARK 2.10. Theorem 2.9 is a generalization of Ekeland's $\varepsilon$-variational principle in [3].

ACKNOWLEDGMENT. This work was supported by KOSEF research project No. 2001-1-10100-005-2.

\section{REFERENCES}

[1] J. Caristi, Fixed point theorems for mappings satisfying inwardness conditions, Trans. Amer. Math. Soc. 215 (1976), 241-251.

[2] L. Ćirić, Semi-continuous mappings and fixed point theorems in quasi-metric spaces, Publ. Math. Debrecen 54 (1999), no. 3-4, 251-261.

[3] I. Ekeland, Nonconvex minimization problems, Bull. Amer. Math. Soc. (N.S.) 1 (1979), no. 3, 443-474.

[4] W. Takahashi, Minimization theorems and fixed point theorems, Nonlinear Analysis and Mathematical Economics (T. Maruyama, ed.), vol. 829, (1993), pp. 175-191.

JeONG Sheok Ume: DePartment of ApPlied MATHematics, Changwon NATional UniverSITY, CHANGWON 641-773, KOREA

E-mail address: jsume@sarim.changwon.ac.kr 


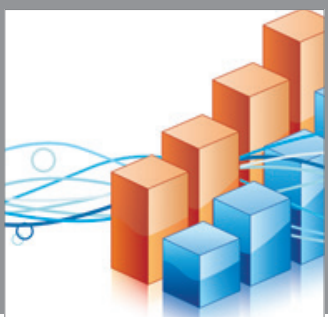

Advances in

Operations Research

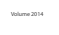

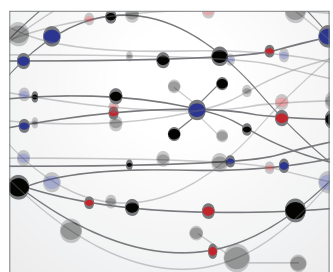

\section{The Scientific} World Journal
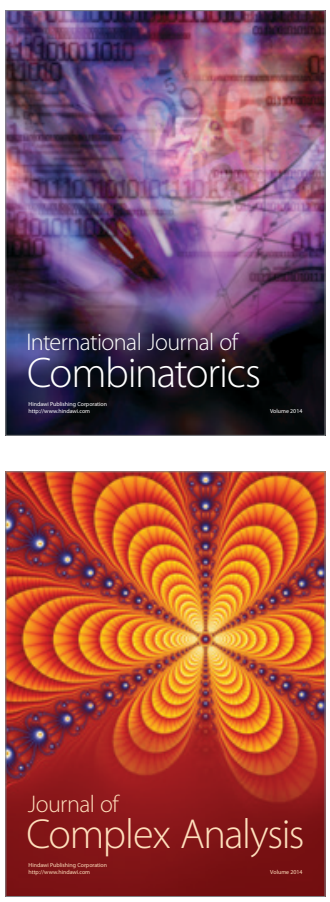

International Journal of

Mathematics and

Mathematical

Sciences
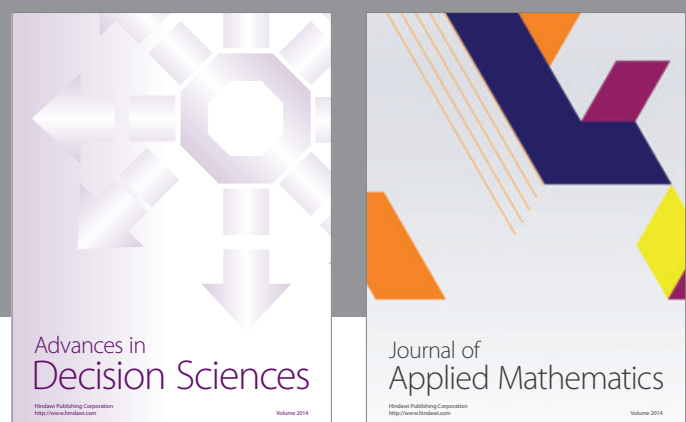

Journal of

Applied Mathematics
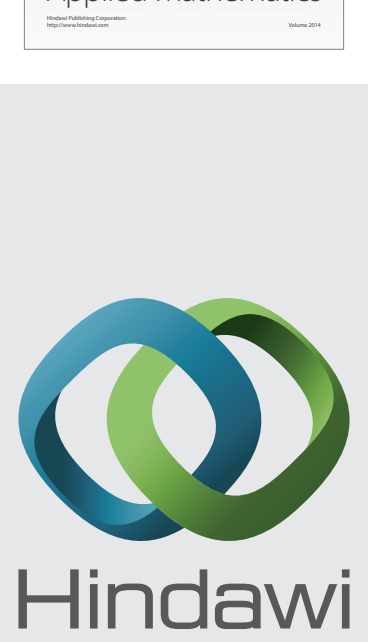

Submit your manuscripts at http://www.hindawi.com
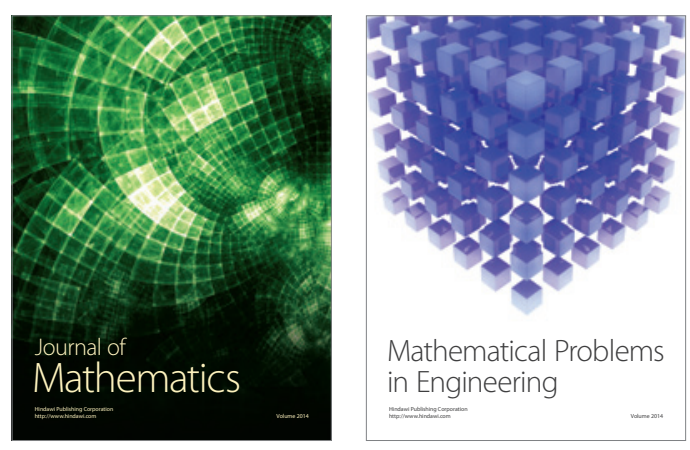

Mathematical Problems in Engineering
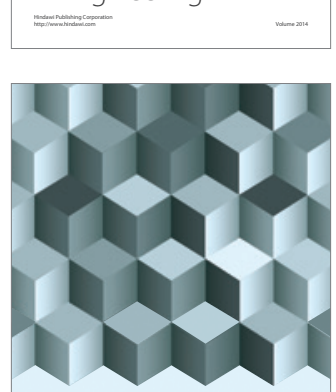

Journal of

Function Spaces
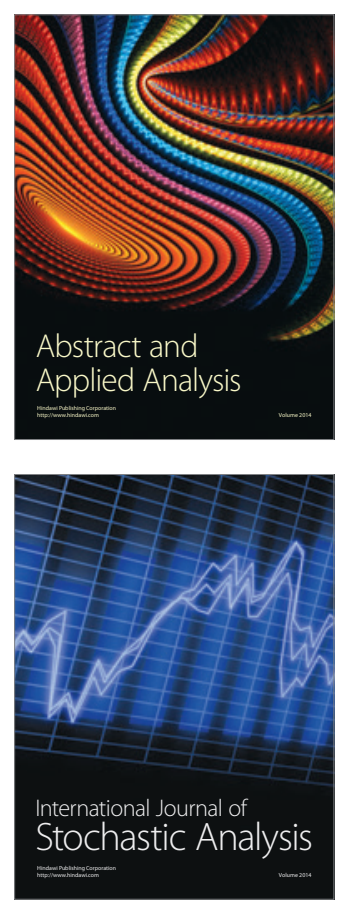

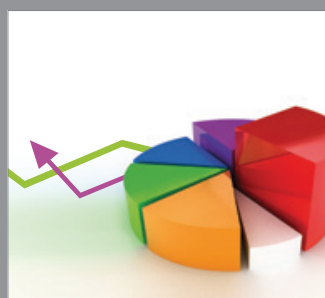

ournal of

Probability and Statistics

Promensencen
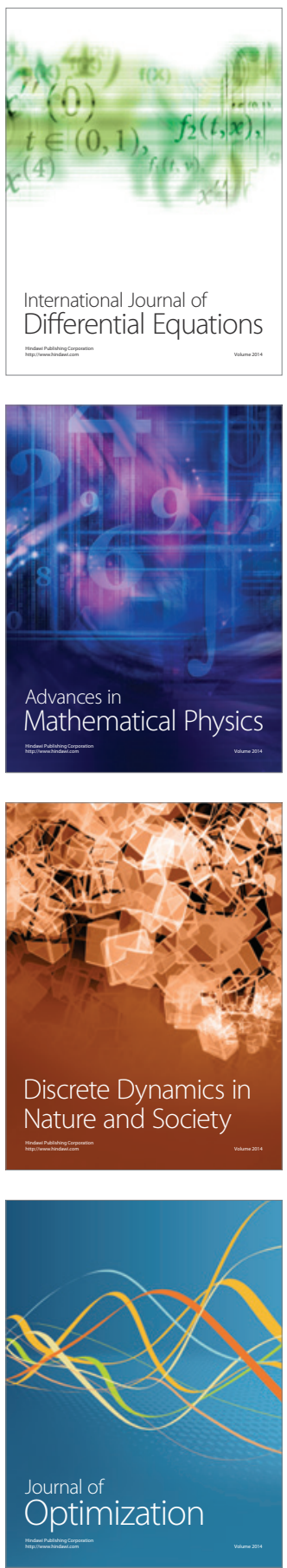